\title{
Survival benefit and toxicity profile of adjuvant icotinib for patients with EGFR mutation-positive non-small cell lung carcinoma: a retrospective study
}

\author{
Ziqing Zeng ${ }^{1,2,3,4 \#}$, Bo Yan ${ }^{2,5 \#}$, Yulong Chen ${ }^{2,5,6 \#}$, Lianmin Zhang ${ }^{2,6,7}$, Jianquan Zhu ${ }^{2,6,7}$, Fan Yang ${ }^{1,2,3,4,8}$, \\ Feng Wei ${ }^{1,2,3,}$, Terence Chi Chun Tam ${ }^{9}$, Diego Kauffmann-Guerrero ${ }^{10}$, Ross Andrew Soo ${ }^{11}$, \\ Xiubao Ren ${ }^{1,2,3,4,8}$, Jian You ${ }^{2,6,7}$
}

${ }^{1}$ Department of Immunology, Tianjin Medical University Cancer Institute and Hospital, National Clinical Research Center for Cancer, Tianjin, China; ${ }^{2}$ Key Laboratory of Cancer Prevention and Therapy, Tianjin, China; ${ }^{3}$ Tianjin's Clinical Research Center for Cancer, Tianjin, China; ${ }^{4}$ Tianjin Key Laboratory of Cancer, Immunology, and Biotherapy, Tianjin, China; ${ }^{5}$ Department of Radiotherapy, Tianjin Medical University Cancer Institute and Hospital, National Clinical Research Center for Cancer, Tianjin, China; ${ }^{6}$ Department of Lung Cancer, Tianjin Medical University Cancer Institute and Hospital, National Clinical Research Center for Cancer, Tianjin, China; ${ }^{7}$ Tianjin's Clinical Research Center for Cancer, Tianjin Lung Cancer Center, Tianjin, China; ${ }^{8}$ Department of Biotherapy, Tianjin Medical University Cancer Institute and Hospital, Tianjin, China; ${ }^{9}$ Department of Medicine, The University of Hong Kong, Queen Mary Hospital, Hong Kong, China; ${ }^{10}$ Division of Respiratory Medicine and Thoracic Oncology, Department of Internal Medicine V and Thoracic Oncology Centre Munich, University of Munich (LMU), Comprehensive Pneumology Center, Member of the German Center for Lung Research (DZL), Munich, Germany; ${ }^{11}$ Department of Haematology-Oncology, National University Cancer Institute Singapore, National University Health System, Singapore, Singapore

Contributions: (I) Conception and design: J You, Y Chen; (II) Administrative support: J You, X Ren; (III) Provision of study materials or patients: Z Zeng, F Yang, F Wei, X Ren; (IV) Collection and assembly of data: Z Zeng, J You; (V) Data analysis and interpretation: Z Zeng, B Yan, Y Chen, L Zhang, J Zhu; (VI) Manuscript writing: All authors; (VII) Final approval of manuscript: All authors.

\#These authors contributed equally to this work.

Correspondence to: Jian You. Key Laboratory of Cancer Prevention and Therapy, Tianjin, China; Department of Lung Cancer, Tianjin Medical University Cancer Institute and Hospital, National Clinical Research Center for Cancer, Tianjin, China; Tianjin's Clinical Research Center for Cancer, Tianjin Lung Cancer Center, Tianjin, China. Email: youjiancn@126.com; Xiubao Ren. Department of Immunology, Tianjin Medical University Cancer Institute and Hospital, National Clinical Research Center for Cancer, Tianjin, China; Key Laboratory of Cancer Prevention and Therapy, Tianjin, China; Tianjin's Clinical Research Center for Cancer, Tianjin, China; Tianjin Key Laboratory of Cancer, Immunology, and Biotherapy, Tianjin, China; Department of Biotherapy, Tianjin Medical University Cancer Institute and Hospital, Tianjin, China. Email: renxiubao@tjmuch.com.

Background Adjuvant epidermal growth factor receptor (EGFR) tyrosine kinase inhibitors (TKIs) are increasing considered for the tailored management of resectable non-small cell lung cancer (NSCLC). This study aimed to analyze the survival and toxicity profile of patients with EGFR mutation-positive NSCLC treated with adjuvant icotinib.

Methods: This was a single-center retrospective study of patients with EGFR mutation-positive NSCLC who underwent R0 (microscopically margin-negative) resection and received adjuvant icotinib between November 2011 and December 2017. The outcomes included 2-year disease-free survival (DFS) rate, 3-year overall survival (OS) rates, DFS, OS, and adverse events (AEs).

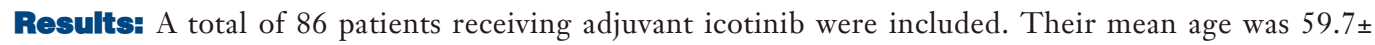
10.0 years, and $26(30.2 \%)$ patients were male. The 2-year DFS rate was $86.7 \%$, and the 3 -year OS rate was $95.3 \%$ with adjuvant icotinib. DFS $(\mathrm{P}=0.044)$ and $\mathrm{OS}(\mathrm{P}=0.003)$ are better in stage I/II disease than in stage III disease. There seems no differences in DFS and OS between patients with low or high preoperative CEA levels (cutoff of $5 \mathrm{ng} / \mathrm{mL}$ ), patients with exon 19 or 21 EGFR mutation or patients with or without smoking history. The most common AEs with adjuvant icotinib were rash (83.7\%) and diarrhea (19.8\%). One (1.2\%) patient-reported grade $\geq 3$ AEs. No treatment-related death occurred.

Conclusions: For patients with EGFR mutation-positive NSCLC, adjuvant icotinib might be associated with a promising survival benefit, with an acceptable toxicity profile. 


\begin{abstract}
Keywords: Non-small cell lung cancer (NSCLC); epidermal growth factor receptor mutation (EGFR mutation); icotinib; adjuvant
\end{abstract}

Submitted Sep 14, 2020. Accepted for publication Dec 09, 2020.

doi: $10.21037 /$ tlcr-20-1214

View this article at: http://dx.doi.org/10.21037/tlcr-20-1214

\section{Introduction}

Non-small cell lung cancer (NSCLC) accounts for 85-90\% of all lung cancers (1). In the United States, the annual incidence of NSCLC is 75 per 100,000 men and 53.5 per 100,000 women; mortality is 55.9 per 100,000 men and 36.3 per 100,000 women (2). In China, the age-standardized incidence of NSCLC is 190.63 per 100,000 individuals, and the age-standardized mortality is 106.98 per 100,000 individuals (3).

The management of early NSCLC requires a multidisciplinary approach (4) with complete surgical resection being the mainstay of treatment (5-8). Whilst platinum based adjuvant chemotherapy improves overall survival (OS) in stage II-IIIA (9-15), treatment related toxicities can affect the quality of life and long-term survival benefits are often minimal (16-18).

More recently, the use of adjuvant epidermal growth factor receptor $(E G F R)$ tyrosine kinase inhibitor (TKI) such as gefitinib, erlotinib or osimertinib, was reported to improve progression free survival in resected NSCLC harboring mutations in EGFR exon 19 and 21, and its use is associated with easier administration and a more favorable toxicity profiles when compared to chemotherapy (19-22). For example, the ADJUVANT/CTONG1104 trial showed for the first time EGFR-TKI adjuvant therapy resulted in a higher 3-year disease-free survival (DFS) rate compared with cisplatin-based adjuvant chemotherapy in patients with completely resected stage II-IIIA (N1-N2) EGFR mutation-positive NSCLC (39.6\% vs. $32.5 \%$; $\mathrm{P}=0.316$ ) (23). However, a recent meta-analysis revealed a PFS benefit of gefitinib or erlotinib compared with chemotherapy in patients with EGFR mutation-positive, but there was no OS benefit (24). A retrospective study showed that, compared with no EGFR-TKI treatment, adjuvant erlotinib or gefitinib can improve the 2-year DFS rate of patients with resected lung adenocarcinoma harboring EGFR exon 19 or 21 mutations ( $89 \%$ vs. $72 \%$; $\mathrm{P}=0.06$ ) (21). In September 2020, the latest results of ADAURA phase 3 trial of osimertinib vs. placebo after resection of non-squamous IBIIIA NSCLC was reported (20). The results showed that the 2-year DFS rate of osimertinib group were higher than that of the placebo control group (89\% vs. $52 \%)$; and the DFS hazard ratio (HR) of the two groups was $0.20(99.12 \%$ CI: 0.14-0.30; $\mathrm{P}<0.001)$.

For the treatment of advanced NSCLC in secondline setting and beyond icotinib was proven to similarly efficacious but safer than gefitinib $(25,26)$. The recent CONVINCE trial showed that icotinib could be used as a first-line agent for patients with EGFR-positive stage IIIB/ IV NSCLC (27), but there is currently insufficient data for its use in the adjuvant setting. A retrospective study of adjuvant icotinib revealed it has survival benefits in $\mathrm{R} 0$ NSCLC with EGFR mutations with acceptable toxicity (28), but a trial of adjuvant icotinib + chemotherapy $v s$. chemotherapy showed no DFS benefit of adding icotinib to chemotherapy (29). The exploration of such approach is important since adjuvant EGFR-TKIs are considered part of a new era for the tailored management of resectable NSCLC (30); importantly since the benefits of adjuvant EGFR-TKIs may be variable among different patient subset, data are required to refine patient selection in order to maximize the clinical benefit of such approach.

The present study aimed to examine the survival and toxicity profile of patients with EGFR mutation-positive NSCLC treated with adjuvant icotinib. The previous data can provide comparation and reference for our research. Therefore, in view of the difficulty of collecting strictly matched cases, we designed this study without a comparative arm. The results could provide insights into the management of selected patients with NSCLC.

We present the following article in accordance with the STROBE reporting checklist (available at http://dx.doi. org/10.21037/tlcr-20-1214).

\section{Methods}

\section{Study design and patients}

Our study was a retrospective analysis of patients with EGFR mutation-positive NSCLC who underwent R0 (microscopically margin-negative) resection and received 
adjuvant icotinib at the Thoracic Oncology Department of the Tianjin Cancer Hospital between November 2011 and December 2017. ADx-ARMS EGFR Five Mutations Detection Kit (Amoy Diagnostics, Xiamen, China) was used to test EGFR mutations. All patients who received icotinib treatment and included in this study were patients who were EGFR-TKI benefit population (EGFRsensitive mutation-positive). Patients with negative or resistant EGFR mutations (such as T790M mutation) were excluded. The inclusion criteria were: (I) age $\geq 18$ years; (II) pathologically confirmed diagnosis of stage IB-IIIB NSCLC and R0 resection (the following risk factors were required for stage IB patients: vascular invasion, visceral pleura involvement, solid or micropapillary components in invasive adenocarcinoma $\geq 30 \%$, or dissemination within the airway); (III) no previous history of chemotherapy, radiotherapy, or targeted therapy; (IV) icotinib was started within eight weeks postoperatively, and there were no signs of tumor recurrence before starting the adjuvant therapy; (V) adequate functions of the hematological system, liver, and kidney; (VI) Eastern Cooperative Oncology Group (ECOG) performance status of $0-1$; and (VII) postoperative survival $>3$ months.

The exclusion criteria were: (I) history of any cancer other than NSCLC [except for cervical carcinoma in situ, cured basal cell carcinoma, or bladder epithelial tumors (including Ta and Tis)] within 5 years before the adjuvant therapy; (II) history of previous interstitial lung disease, drug-induced interstitial lung disease, radiation pneumonitis requiring steroid therapy, or any clinically documented active interstitial lung disease, or idiopathic pulmonary fibrosis; (III) partially controlled eye inflammation or eye infection, or any condition that may cause the above-mentioned eye diseases; (IV) any unstable systemic disease, including active infection, uncontrolled hypertension, unstable angina, angina that has started within the last 3 months, congestive heart failure (New York Heart Association grade $\geq \mathrm{II}$ ), myocardial infarction (within 6 months), severe arrhythmia, or liver, kidney or metabolic diseases requiring medications; (V) human immunodeficiency virus infection; (VI) pregnant or lactating women; or (VII) history of previous neurological or mental disorders, including epilepsy or dementia.

The demographic and clinical characteristics of all patients were extracted from their medical records. All procedures performed in this study involving human participants were in accordance with the Declaration of Helsinki (as revised in 2013). The Ethics Committee approved this study of the Tianjin Cancer Hospital (No. bc2019078). Adjuvant icotinib was used only after a comprehensive discussion between the patient and the physicians, and after tumor board discussion. The patients provided informed consent before receiving adjuvant icotinib.

\section{Adjuvant therapy}

R0 resection was achieved for all patients. Patients received adjuvant icotinib (125 mg, tid, orally) (Betta Pharmaceuticals Co., Ltd., Zhejiang, China) for 2 years. The medication was withdrawn if there was disease recurrence or intolerable toxicities. Any other postoperative combined therapies were recorded.

\section{Outcomes and follow-up}

Patients were followed routinely every 3 months. The outcomes included the 2-year DFS rate, 3-year OS rates, DFS, OS, and adverse events (AEs). Subgroup DFS and OS analyses were conducted with disease stages, preoperative carcinoembryonic antigen (CEA) levels, history of smoking, and EGFR mutation. DFS was defined as the time from surgery to disease recurrence or all-cause death, whichever occurred first. The OS was defined as the time from surgery to all-cause death. AEs were reported and graded according to the National Cancer Institute (NCI) Common Terminology Criteria for Adverse Events (CTCAE), version 4.0.

\section{Statistical analysis}

SPSS 22.0 (IBM, Armonk, NY, USA) was used for statistical analysis. Continuous variables are expressed as means \pm standard deviation (SD). Categorical variables are expressed as frequency (percentage). Patient DFS and OS were plotted using the Kaplan-Meier curve and compared using the log-ranking test. $\mathrm{P}<0.05$ was considered statistically significant. The minimal post-hoc power was defined as $60 \%$.

\section{Results}

\section{Characteristics of the patients}

Table 1 presents the characteristics of the 86 patients receiving adjuvant icotinib. Their mean age was $59.7 \pm 10.0$ years, and $26(30.2 \%)$ patients were male. All patients had an ECOG performance status of 0 . Most patients had lung adenocarcinoma 
Table 1 Demographic and clinical characteristics

\begin{tabular}{|c|c|}
\hline Variable & Icotinib $(n=86)$ \\
\hline Age (years), mean $\pm S D$ & $59.7 \pm 10.0$ \\
\hline Male, n (\%) & $26(30.2)$ \\
\hline Smoking, $\mathrm{n}(\%)$ & $25(29.1)$ \\
\hline \multicolumn{2}{|l|}{ Histology, n (\%) } \\
\hline Adenocarcinoma & $81(94.2)$ \\
\hline Squamous cell carcinoma & $3(3.5)$ \\
\hline Adenosquamous carcinoma & $1(1.2)$ \\
\hline Signet ring cell carcinoma & $1(1.2)$ \\
\hline \multicolumn{2}{|l|}{ Comorbidity, n (\%) } \\
\hline Hypertension & $28(32.6)$ \\
\hline Coronary heart disease & $7(8.1)$ \\
\hline Diabetes mellitus & $8(9.3)$ \\
\hline Myocardial ischemia & $5(5.8)$ \\
\hline Hypothyroidism & $4(4.7)$ \\
\hline \multicolumn{2}{|l|}{ Type of resection, n (\%) } \\
\hline Lobectomy & $67(77.9)$ \\
\hline Bilobectomy & $15(17.4)$ \\
\hline Segmentectomy & $1(1.2)$ \\
\hline Sleeve pneumonectomy & $2(2.3)$ \\
\hline Total pneumonectomy & $1(1.2)$ \\
\hline \multicolumn{2}{|l|}{ TNM stage, n (\%) } \\
\hline IB & $46(53.5)$ \\
\hline IIA & $3(3.5)$ \\
\hline IIB & $5(5.8)$ \\
\hline IIIA & $24(27.9)$ \\
\hline IIIB & $8(9.3)$ \\
\hline \multicolumn{2}{|l|}{ T stage, $\mathrm{n}(\%)$} \\
\hline $\mathrm{T} 1$ & $1(1.2)$ \\
\hline $\mathrm{T} 2$ & $69(80.2)$ \\
\hline T3 & $6(7.0)$ \\
\hline $\mathrm{T} 4$ & $10(11.6)$ \\
\hline \multicolumn{2}{|l|}{ N stage, n (\%) } \\
\hline NO & $57(66.3)$ \\
\hline N1 & $2(2.3)$ \\
\hline N2 & $27(31.4)$ \\
\hline
\end{tabular}

Table 1 (continued)

\begin{tabular}{lc}
\hline Variable & Icotinib $(\mathrm{n}=86)$ \\
\hline Preoperative CEA $(\mathrm{ng} / \mathrm{mL}), \mathrm{n}(\%)$ & $63(73.2)$ \\
$\leq 5$ & $22(25.6)$ \\
$>5$ & $1(1.2)$ \\
Unknown & \\
EGFR mutation, $\mathrm{n}(\%)$ & $2(2.3)$ \\
Exon 18 & $40(46.5)$ \\
Exon 19 & $1(1.2)$ \\
Exon 20 & $42(48.8)$ \\
Exon 21 & $1(1.2)$ \\
Exon 19 and 21 & \\
Postoperative CEA (ng/ml), $\mathrm{n}(\%)$ & $69(80.2)$ \\
$\leq 5$ & $6(7.0)$ \\
$>5$ & $11(12.8)$ \\
Unknown & \\
Postoperative combined treatment, $\mathrm{n}$ & \\
(\%) & \\
Chemotherapy & \\
Radiotherapy & $(5.8)$ \\
\hline
\end{tabular}

CEA, carcinoembryonic antigen; EGFR, epidermal growth factor receptor; $\mathrm{SD}$, standard deviation.

[81 (94.2\%)], T2 disease [69 (80.2\%)], N0 disease [57 (66.3\%)], preoperative CEA $\leq 5 \mathrm{ng} / \mathrm{mL}$ [63 (73.2\%)], and underwent lobectomy [67 (77.9\%)]. The EGFR mutations were: exon 18 $(\mathrm{n}=2,2.3 \%)$, exon $19(\mathrm{n}=40,46.5 \%)$, exon $20(\mathrm{n}=1,1.2 \%)$, exon $21(\mathrm{n}=42,48.8 \%)$, and compound exon $19 \& 21$ mutation $(\mathrm{n}=1$, $1.2 \%)$. In addition to adjuvant icotinib, 5 (5.8\%) and $13(15.1 \%)$ patients received postoperative chemotherapy and radiotherapy, respectively.

\section{Survival}

The median follow-up time was 48 months. Most patients (93\%) had a follow-up period of more than 3 years. Figure 1 presents the survival data of patients receiving adjuvant icotinib. The median DFS and OS were not reached. The 2 -year DFS rate and the 3 -year OS rate was $86.7 \%$ and $95.3 \%$, respectively.

Table 1 (continued) 

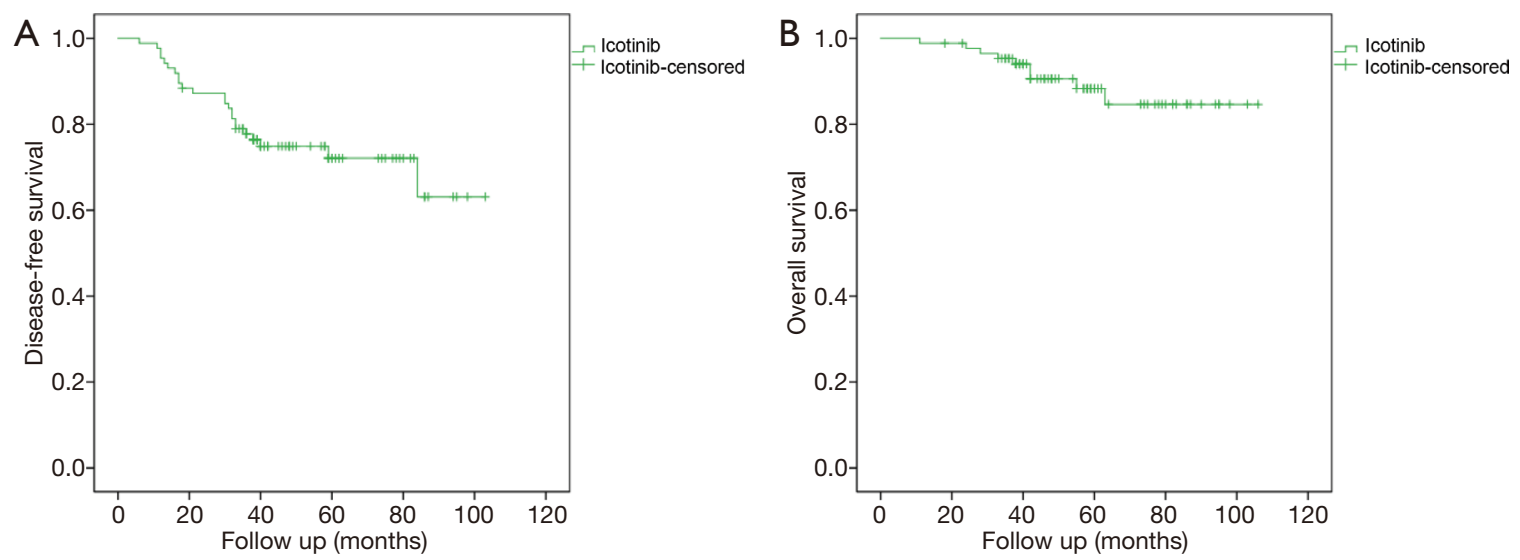

Figure 1 Kaplan-Meier curves of patients receiving adjuvant icotinib. (A) Disease-free survival. (B) Overall survival.

\section{Subgroup analysis}

We excluded patients who had received combined postoperative therapy and performed a subgroup analysis. The results showed that DFS $(\mathrm{P}=0.044)$ and $\mathrm{OS}(\mathrm{P}=0.003)$ is better in stage I/II disease than in stage III disease (Figure 2). Specifically, DFS $(\mathrm{P}=0.041)$ and $\mathrm{OS}(\mathrm{P}=0.007)$ is better in T1-2 disease than in T3-4 disease. N0 disease had better DFS $(\mathrm{P}=0.016)$, and a better trend of OS $(\mathrm{P}=0.061)$ was found compared with N1-2 disease (Figure S1). There seems no differences in DFS and OS between patients with low or high preoperative CEA levels (cutoff of $5 \mathrm{ng} / \mathrm{mL}$ ), patients with exon 19 or 21 EGFR mutation or patients with or without smoking history (Figure 2). However, as some subgroup numbers are small (especially after we excluded patients with combined treatment), we calculated the posthoc power of all subgroup analyses, and the power is low $(<60 \%)$. Thus, we cannot draw the conclusions that there is no difference in DFS or OS between those groups as this study are not powered to answer. However, all these statistical results in this study may provide some hints for future research, and these results need to be verified by future studies with larger sample sizes.

\section{AEs}

AEs are shown in Table 2. Of the patients who received adjuvant icotinib, 73 (84.9\%) had grade 1-2 AEs, and one (1.2\%) had grade 3 AEs. Among them, 72 (83.7\%) had a rash, 17 (19.8\%) developed diarrhea, 4 (4.7\%) had elevated transaminases, 5 (5.8\%) experienced fatigue (including one grade 3 fatigue), 4 (4.7\%) had oral ulcers, and 1 $(1.2 \%)$ had nausea. Most AEs were relieved without any treatment. Patients without spontaneous relief were treated symptomatically, and no severe AEs, including interstitial lung disease, occurred. Multiple AEs were observed in 24 (32.9\%) patients.

\section{Discussion}

EGFR-TKIs have been approved by Food and Drug Administration and Chinese National Medical Products Administration for use in the treatment of advanced NSCLC patients who cannot be surgically resected, and the efficacy of EGFR-TKIs were usually better than that of chemotherapy or no treatment (31). A meta-analysis showed that, in advanced NSCLC, the median survival time was $13.26,13.52$, and 12.58 months for gefitinib, erlotinib and icotinib, respectively (25). Icotinib has similar efficacy than gefitinib but a more favorable safety profile when used as a second- or further-line therapy in patients with advanced NSCLC $(25,26)$, but there is currently insufficient data for icotinib as adjuvant therapy in patients with EGFR mutation-positive NSCLC (27). EGFR-TKIs are considered part of a new era for the tailored management of resectable EGFR mutation-positive NSCLC (30), and our results suggest that the use of adjuvant icotinib in such cohort might be associated with a promising survival benefit with an acceptable toxicity profile.

It has been well documented that adjuvant platinum based chemotherapy improves the survival in patients with resected NSCLC (9-12), but chemotherapy-related AEs and treatment related mortality were reported (9). More recently, adjuvant gefitinib, erlotinib and osimertinib have shown improvement in PFS in resected stage I-III NSCLC with EGFR exon 19 and 21 mutations (20-22). Compared 


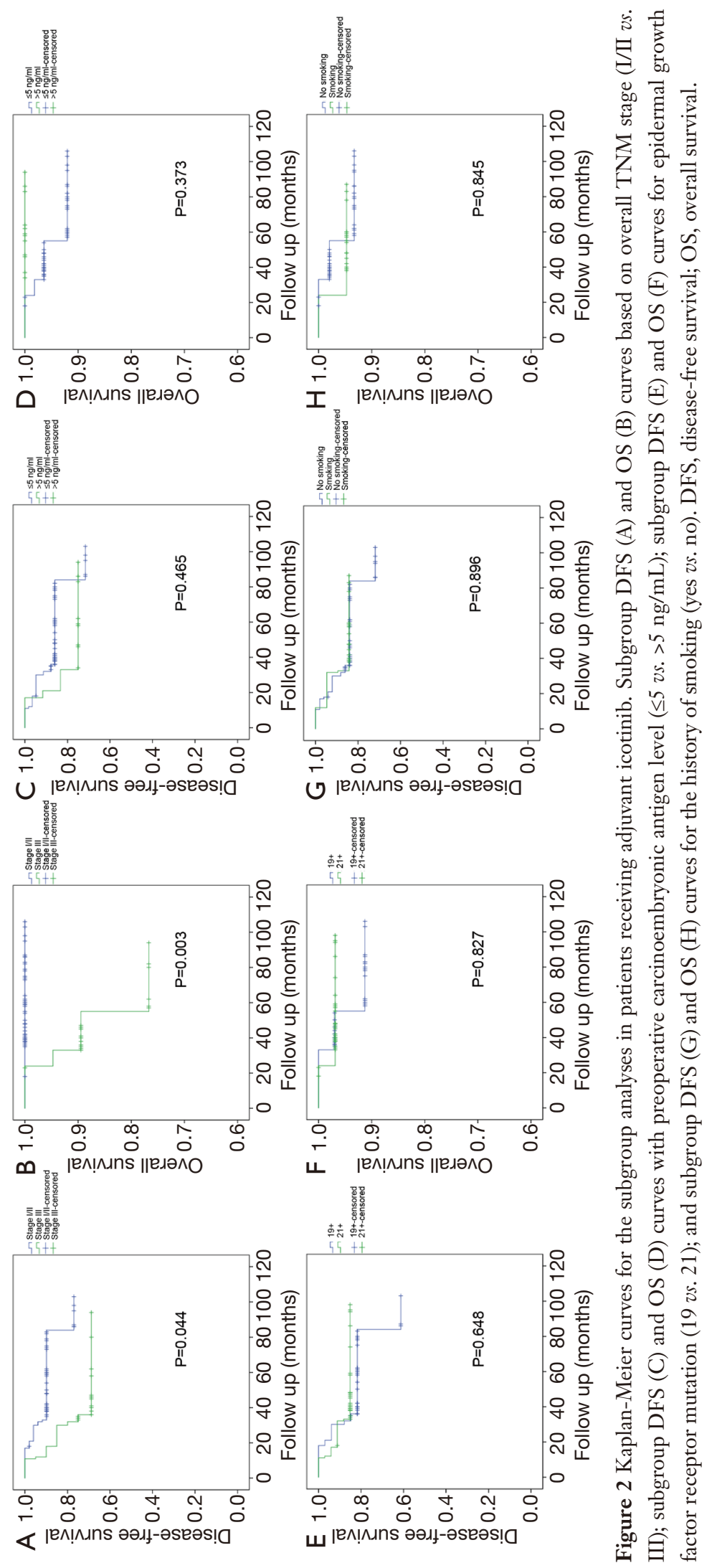


Table 2 Adverse events

\begin{tabular}{lccc}
\hline \multirow{2}{*}{ The event and number (\%) } & \multicolumn{3}{c}{ Icotinib (n=86) } \\
\cline { 2 - 4 } & Grades 1-2 & Grade 3 & Grade 4 \\
\hline Any adverse event & $73(84.9)$ & $1(1.2)$ & 0 \\
Rash & $72(83.7)$ & 0 & 0 \\
Diarrhea & $17(19.8)$ & 0 & 0 \\
Fatigue & $4(4.7)$ & $1(1.2)$ & 0 \\
Elevated ALT/AST & $4(4.7)$ & 0 & 0 \\
Oral ulcers & $4(4.7)$ & 0 & 0 \\
Nausea & $1(1.2)$ & 0 & 0 \\
\hline
\end{tabular}

ALT, alanine aminotransferase; AST, aspartate aminotransferase.

with gefitinib, icotinib has similar efficacy, but a better safety profile $(25,26)$, and the CONVINCE trial showed better outcomes of the first-line icotinib vs. chemotherapy for stage IIIB-IV NSCLC (27). A retrospective study of adjuvant icotinib revealed it has survival benefits in $\mathrm{R} 0$ NSCLC with EGFR mutations with acceptable toxicity (28). In most EGFR adjuvant trials, there is the problem of the lacking OS data as well the problem regarding the treatment duration. The lack of OS data may be due to insufficient follow-up time and insufficient number of events, in which condition, OS data may be unreliable therefore have not been displayed. Follow-up data of these clinical trials can be collected and updated in the future. At ASCO 2020, the OS data of ADJUVANT was revealed, showing that the median OS of patients receiving adjuvant gefitinib was 75.5 months, which was nearly 13 months longer than the 62.8 months in the adjuvant chemotherapy group (HR: 0.92; $95 \%$ CI: $0.62-1.36 ; \mathrm{P}=0.674$ ) (32). Treatment duration is also an issue that have not been concluded. Current EGFR-TKI adjuvant trials differ in the choice of treatment duration. Regarding the exploration of treatment duration, some studies have been carried out, we look forward to the results of those studies. In present study, two years was adopted as treatment duration and the DFS/OS data was shown.

The present study showed that the 2-year DFS rate was $86.7 \%$ and the 3 -year OS rate was $95.3 \%$, which are better than the historical survival data with chemotherapy (2-year DFS of $57-76 \%$, 3-year OS of $62-74 \%$ ) or with no treatment (2-year DFS of $47-60 \%, 3$-year OS of $57-72 \%$ ) (9-12), suggesting a possible better survival benefit for adjuvant icotinib targeted therapy compared with adjuvant chemotherapy. The median DFS and OS were not reached in the present study. These results might be associated with excellent baseline characteristics of the patients since about $50 \%$ of the patients were stage IB, and the ECOG performance status was 0 in all patients. These findings suggest that adjuvant therapy with icotinib is feasible and might result in an excellent prognosis in patients with stage IB-IIIB NSCLC.

Further subgroup analysis showed that both DFS and OS were better for stage I/II disease than for stage III and T1-2 disease compared with T3-4. DFS was better for N0 tumors than for N1-2. Those results are in agreement with the recognized prognostic factors of NSCLC $(4,8)$.

In the present study, the most common AEs in the icotinib group were rash and diarrhea, with acceptable tolerability. Spontaneous relief was observed in most patients, and no severe AEs, including interstitial lung disease, occurred. If interstitial pneumonia occurs, the treatment mainly includes: stop taking EGFR-TKI immediately; clinical treatment strategies are mainly symptomatic and supportive treatment: oxygen inhalation, anti-inflammatory, anti-infective, and anti-fibrotic treatments. Previous studies of icotinib already revealed its favorable safety profile (25-27,33). However, in terms of some specific side effects, some other EGFR-TKIs may have less side effects. For example, clinical trials $(27,34,35)$ showed that although icotinib has the lowest overall incidence of diarrhea, many other EGFR-TKIs have a lower incidence of grade $\geq 3$ diarrhea. In addition, compared with icotinib, osimertinib has a lower incidence of druginduced liver injury, gefitinib and osimertinib have a lower incidence of grade $\geq 3$ rash, etc. The results of this study suggest icotinib with a standard dose is suitable for adjuvant therapy in patients with R0 resected NSCLC, but further prospective clinical studies must validate this suitability. In clinical practice, we need to select the most suitable EGFRTKI according to the specific situation.

This study has limitations. First, biases are unavoidable in single-center retrospective studies. In the future, we will actively carry out multi-center clinical study in order to obtain more generalized results. Second, multivariate analysis was statistically impractical due to the high censoring rate. Third, this was a single-arm study without a comparator. Furthermore, some issues need to be addressed: (I) whether icotinib monotherapy is enough; (II) the optimal duration of adjuvant icotinib therapy; (III) sequence and timing of adjuvant targeted therapy and chemotherapy; and (IV) optimal target patients. The present study indicated that adjuvant icotinib therapy may prolonged survival with 
an acceptable safety profile, Further randomized controlled trials must verify the efficacy and safety of adjuvant therapy with icotinib to support our hypothesis. These trials might further confirm the impact of adjuvant icotinib therapy on DFS and OS and will identify the target population.

\section{Conclusions}

This retrospective study suggests that adjuvant icotinib might be associated with a promising survival benefit with an acceptable toxicity profile. The OS rate observed in this study was higher than that reported in the literature for chemotherapy. These results are essential because EGFR-TKIs are considered central to the modern tailored management of resectable NSCLC.

\section{Acknowledgments}

The authors sincerely appreciate all the physicians and patients who participated in this study, and also appreciate the academic support from AME Lung Cancer Collaborative Group.

Funding: None.

\section{Footnote}

Reporting Checklist: The authors have completed the STROBE reporting checklist. Available at http://dx.doi. org/10.21037/tlcr-20-1214

Data Sharing Statement: Available at http://dx.doi. org/10.21037/tlcr-20-1214

Conflicts of Interest: All authors have completed the ICMJE uniform disclosure form (available at http://dx.doi. org/10.21037/tlcr-20-1214). The authors have no conflicts of interest to declare.

Ethical Statement: The authors are accountable for all aspects of the work in ensuring that questions related to the accuracy or integrity of any part of the work are appropriately investigated and resolved. All procedures performed in this study involving human participants were in accordance with the Declaration of Helsinki (as revised in 2013). The Ethics Committee approved this study of the Tianjin Cancer Hospital (No. bc2019078). The patients provided informed consent before receiving adjuvant icotinib.
Open Access Statement: This is an Open Access article distributed in accordance with the Creative Commons Attribution-NonCommercial-NoDerivs 4.0 International License (CC BY-NC-ND 4.0), which permits the noncommercial replication and distribution of the article with the strict proviso that no changes or edits are made and the original work is properly cited (including links to both the formal publication through the relevant DOI and the license). See: https://creativecommons.org/licenses/by-nc-nd/4.0/.

\section{References}

1. Novello S, Barlesi F, Califano R, et al. Metastatic non-small-cell lung cancer: ESMO Clinical Practice Guidelines for diagnosis, treatment and follow-up. Ann Oncol 2016;27:v1-v27.

2. Siegel RL, Miller KD, Jemal A. Cancer statistics, 2019. CA Cancer J Clin 2019;69:7-34.

3. de Groot PM, Wu CC, Carter BW, et al. The epidemiology of lung cancer. Transl Lung Cancer Res 2018;7:220-33.

4. NCCN Clinical Practice Guidelines in Oncology (NCCN Guidelines). Non-Small Cell Lung Cancer. Version 3.2019. Fort Washington: National Comprehensive Cancer Network; 2019.

5. Lee GD, Kim DK, Jang SJ, et al. Significance of R1resection at the bronchial margin after surgery for non-small-cell lung cancer. Eur J Cardiothorac Surg 2017;51:176-81.

6. Smeltzer MP, Lin CC, Kong FS, et al. Survival impact of postoperative therapy modalities according to margin status in non-small cell lung cancer patients in the United States. J Thorac Cardiovasc Surg 2017;154:661-672.e10.

7. Predina JD, Keating J, Patel N, et al. Clinical implications of positive margins following non-small cell lung cancer surgery. J Surg Oncol 2016;113:264-9.

8. Osarogiagbon RU, Lin CC, Smeltzer MP, et al. Prevalence, Prognostic Implications, and Survival Modulators of Incompletely Resected Non-Small Cell Lung Cancer in the U.S. National Cancer Data Base. J Thorac Oncol 2016;11:e5-16.

9. Douillard JY, Rosell R, De Lena M, et al. Adjuvant vinorelbine plus cisplatin versus observation in patients with completely resected stage IB-IIIA non-small-cell lung cancer (Adjuvant Navelbine International Trialist Association [ANITA]): a randomised controlled trial. Lancet Oncol 2006;7:719-27.

10. Winton T, Livingston R, Johnson D, et al. Vinorelbine 
plus cisplatin vs. observation in resected non-small-cell lung cancer. N Engl J Med 2005;352:2589-97.

11. Pignon JP, Tribodet H, Scagliotti GV, et al. Lung adjuvant cisplatin evaluation: a pooled analysis by the LACE Collaborative Group. J Clin Oncol 2008;26:3552-9.

12. Group NM-aC, Arriagada R, Auperin A, et al. Adjuvant chemotherapy, with or without postoperative radiotherapy, in operable non-small-cell lung cancer: two meta-analyses of individual patient data. Lancet 2010;375:1267-77.

13. Arriagada R, Bergman B, Dunant A, et al. Cisplatinbased adjuvant chemotherapy in patients with completely resected non-small-cell lung cancer. $\mathrm{N}$ Engl J Med 2004;350:351-60.

14. Butts CA, Ding K, Seymour L, et al. Randomized phase III trial of vinorelbine plus cisplatin compared with observation in completely resected stage IB and II nonsmall-cell lung cancer: updated survival analysis of JBR10. J Clin Oncol 2010;28:29-34.

15. Strauss GM, Herndon JE 2nd, Maddaus MA, et al. Adjuvant paclitaxel plus carboplatin compared with observation in stage IB non-small-cell lung cancer: CALGB 9633 with the Cancer and Leukemia Group B, Radiation Therapy Oncology Group, and North Central Cancer Treatment Group Study Groups. J Clin Oncol 2008;26:5043-51.

16. Bezjak A, Lee CW, Ding K, et al. Quality-of-life outcomes for adjuvant chemotherapy in early-stage nonsmall-cell lung cancer: results from a randomized trial, JBR.10. J Clin Oncol 2008;26:5052-9.

17. Wintner LM, Giesinger JM, Zabernigg A, et al. Quality of life during chemotherapy in lung cancer patients: results across different treatment lines. Br J Cancer 2013;109:2301-8.

18. Ramirez RA, Lu J, Thomas KEH. Quality of life for non-small cell lung cancer patients in the age of immunotherapy. Transl Lung Cancer Res 2018;7:S149-52.

19. Wu JX, He Q, Ye F, et al. EGFR-TKI-based vs nonEGFR-TKI-based adjuvant therapy in resected nonsmall-cell lung cancer with EGFR mutations: a metaanalysis of randomized controlled trials. Onco Targets Ther 2018;11:6803-10.

20. Wu YL, Tsuboi M, He J, et al. Osimertinib in Resected EGFR-Mutated Non-Small-Cell Lung Cancer. N Engl J Med 2020;383:1711-23.

21. Janjigian YY, Park BJ, Zakowski MF, et al. Impact on disease-free survival of adjuvant erlotinib or gefitinib in patients with resected lung adenocarcinomas that harbor EGFR mutations. J Thorac Oncol 2011;6:569-75.

22. Pennell NA, Neal JW, Chaft JE, et al. SELECT: A Phase II Trial of Adjuvant Erlotinib in Patients With Resected Epidermal Growth Factor Receptor-Mutant Non-SmallCell Lung Cancer. J Clin Oncol 2019;37:97-104.

23. Zhong WZ, Wang Q, Mao WM, et al. Gefitinib versus vinorelbine plus cisplatin as adjuvant treatment for stage II-IIIA (N1-N2) EGFR-mutant NSCLC (ADJUVANT/ CTONG1104): a randomised, open-label, phase 3 study. Lancet Oncol 2018;19:139-48.

24. Lee CK, Davies L, Wu YL, et al. Gefitinib or Erlotinib vs Chemotherapy for EGFR Mutation-Positive Lung Cancer: Individual Patient Data Meta-Analysis of Overall Survival. J Natl Cancer Inst 2017;109. doi: 10.1093/jnci/ djw279.

25. Liu Y, Zhang Y, Feng G, et al. Comparison of effectiveness and adverse effects of gefitinib, erlotinib and icotinib among patients with non-small cell lung cancer: A network meta-analysis. Exp Ther Med 2017;14:4017-32.

26. Shi $Y$, Zhang L, Liu X, et al. Icotinib versus gefitinib in previously treated advanced non-small-cell lung cancer (ICOGEN): a randomised, double-blind phase 3 noninferiority trial. Lancet Oncol 2013;14:953-61.

27. Shi YK, Wang L, Han BH, et al. First-line icotinib versus cisplatin/pemetrexed plus pemetrexed maintenance therapy for patients with advanced EGFR mutation-positive lung adenocarcinoma (CONVINCE): a phase 3, open-label, randomized study. Ann Oncol 2017;28:2443-50.

28. Yao S, Zhi X, Wang R, et al. Retrospective study of adjuvant icotinib in postoperative lung cancer patients harboring epidermal growth factor receptor mutations. Thorac Cancer 2016;7:543-8.

29. Feng S, Wang Y, Cai K, et al. Randomized Adjuvant Chemotherapy of EGFR-Mutated Non-Small Cell Lung Cancer Patients with or without Icotinib Consolidation Therapy. PLoS One 2015;10:e0140794.

30. Shen P, Zhong W. Adjuvant EGFR TKI therapy for resectable non-small cell lung cancer: new era for personalized medicine. J Thorac Dis 2018;10:1364-9.

31. Yoon SK, Choi CM, Lee JC. The continued EGFRTKI with cytotoxic chemotherapy at progression-poison or medicine? Transl Lung Cancer Res 2018;7:S176-8.

32. Wu YL, Zhong WZ, Wang Q, et al. CTONG1104: Adjuvant gefitinib versus chemotherapy for resected 
N1-N2 NSCLC with EGFR mutation-Final overall survival analysis of the randomized phase III trial 1 analysis of the randomized phase III trial. J Clin Oncol 2020;38:abstr 9005.

33. Biaoxue R, Hua L, Wenlong G, et al. Efficacy and safety of icotinib in treating non-small cell lung cancer: a systematic evaluation and meta-analysis based on 15 studies. Oncotarget 2016;7:86902-13.

Cite this article as: Zeng Z, Yan B, Chen Y, Zhang L, Zhu J, Yang F, Wei F, Tam TCC, Kauffmann-Guerrero D, Soo RA, Ren X, You J. Survival benefit and toxicity profile of adjuvant icotinib for patients with EGFR mutation-positive non-small cell lung carcinoma: a retrospective study. Transl Lung Cancer Res 2020;9(6):2401-2410. doi: 10.21037/tlcr-20-1214
34. Mok TS, Wu YL, Ahn MJ, et al. Osimertinib or Platinum-Pemetrexed in EGFR T790M-Positive Lung Cancer. N Engl J Med 2017;376:629-40.

35. Mok TS, Wu YL, Thongprasert S, et al. Gefitinib or carboplatin-paclitaxel in pulmonary adenocarcinoma. $\mathrm{N}$ Engl J Med 2009;361:947-57.

(English Language Editor: J. Chapnick) 


\section{Supplementary}
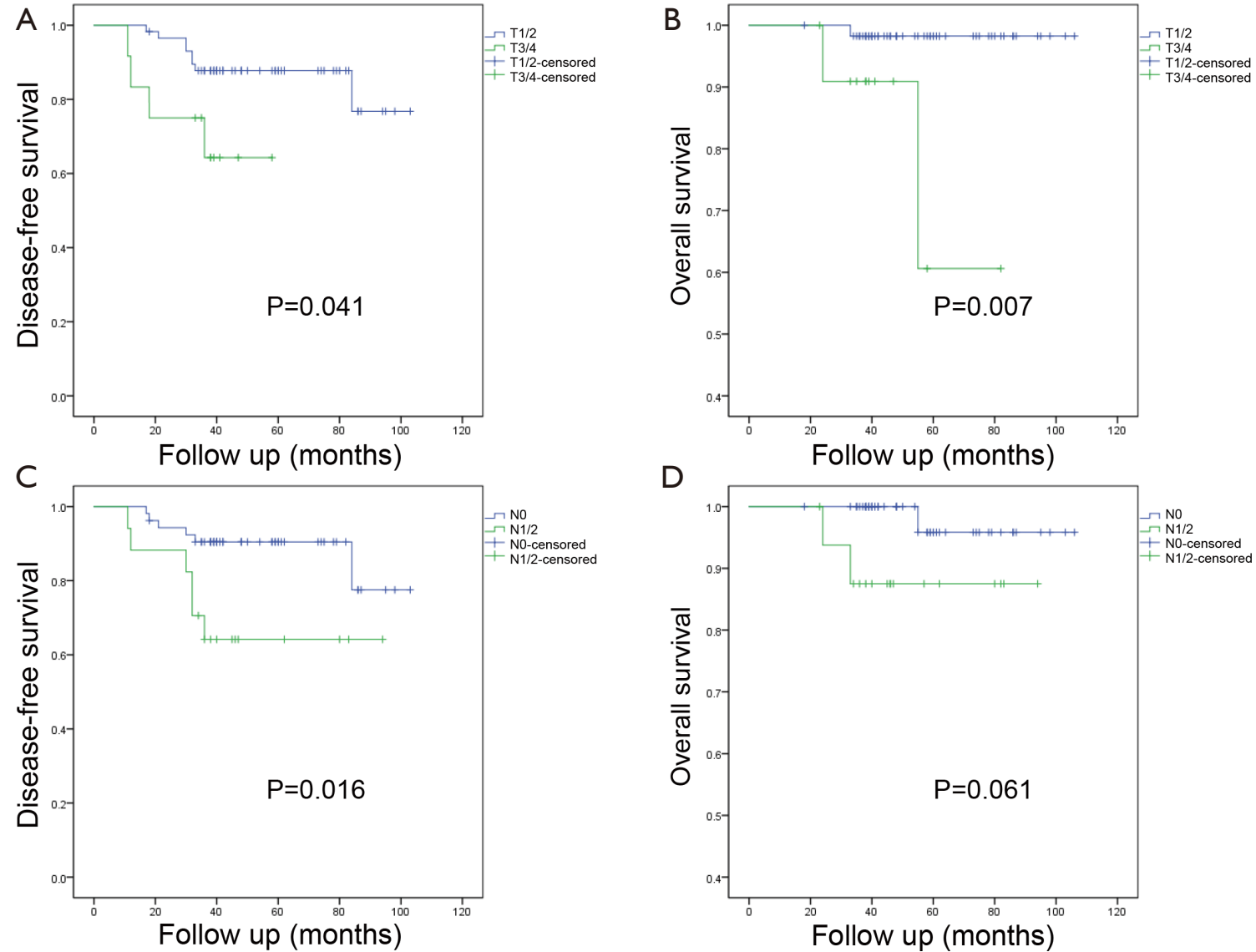

Figure S1 Kaplan-Meier curves for the subgroup analyses in patients receiving adjuvant icotinib. Subgroup DFS (A) and OS (B) curves with T stage (T1/2 vs. T3/4); and subgroup DFS (C) and OS (D) curves with N stage (N0 vs. N1/2). DFS, disease-free survival; OS, overall survival. 